\title{
Immunostimulatory effects of asiatic acid and madecassoside on the phagocytosis activities of macrophages cell line (J774A.1)
}

\author{
Nurul Hikmah Harun ${ }^{*}$, Wan Amir Nizam Wan Ahmad², Rapeah Suppian ${ }^{2}$ \\ ${ }^{1}$ School of Biomedicine, Faculty of Health Sciences, University of Sultan Zainal Abidin, Kuala Nerus, Terengganu, Malaysia. \\ ${ }^{2}$ School of Health Sciences, Health Campus, University of Science Malaysia, Kubang Kerian, Kelantan, Malaysia.
}

\begin{tabular}{l}
\hline ARTICLE INFO \\
\hline Received on: $25 / 05 / 2021$ \\
Accepted on: $28 / 7 / 2021$ \\
Available online: $03 / 11 / 2021$
\end{tabular}

\section{Key words:}

Asiatic acid, madecassoside, phagocytosis, nitric oxide, iNOS, macrophage J774A.1.

\begin{abstract}
Macrophages play a vital role in primary immune responses mainly by carrying out phagocytosis that involves the production of nitric oxide (NO). NO is synthesized by inducible nitric oxide synthase (iNOS) to combat aetiological agents that are able to cause infectious diseases (IDs). Asiatic acid (AA) and madecassoside (MA) are two pentacyclic triterpene compounds derived from Centella asiatica (Linn.) Urban that involved in many pharmacological activities of this plant. However, there is still limited study using a normal immune model that resembles a healthy community to test the immunomodulatory effect of these compounds. Thus, this study used unstimulated macrophages as an in vitro normal model to investigate the effect of both compounds on phagocytosis activity. To achieve the aim of this study, the effects of AA and MA, alone and in combination, on the phagocytosis uptake, NO production, and iNOS expression of macrophages after 24 hours of treatment were measured. Briefly, macrophages (J774A.1) were cultured in five groups, namely untreated macrophages, macrophages treated with lipopolysaccharides (LPS) as a positive control, and macrophages treated with AA and MA alone and in combination (AA + MA). The investigation on phagocytosis uptake, NO production, and iNOS expression were conducted by using microscopic analysis on Giemsastained slides, the Griess assay, and Western blot, respectively. The results presented that the combination (AA + MA) treatment enhanced phagocytosis uptake, NO production, and iNOS expression of macrophages when compared to the untreated macrophages as well as macrophages treated with AA and MA, respectively. In conclusion, the combination $(\mathrm{AA}+\mathrm{MA})$ treatment is able to stimulate phagocytosis activity of macrophages and this new finding provides initial knowledge for the development of a natural product-based preventive agent against IDs.
\end{abstract}

\section{INTRODUCTION}

According to the World Health Organization, infectious diseases (IDs) are of the highest concern among healthcareproviding organizations, mainly in developing countries (WHO, 2000). It is mainly managed through preventive measures including vaccination as well as using suitable synthetic medicinal drugs, such as antibiotics and antiviral drugs, to treat existing diseases (Chee et al., 2017; Maslow, 2017). However, antibiotics have many serious adverse effects such as allergic reactions and removal of normal flora bacteria in the body (Glick, 2016).

\footnotetext{
${ }^{*}$ Corresponding Author

Nurul Hikmah Harun, School of Biomedicine, Faculty of Health Sciences, University of Sultan Zainal Abidin, Kuala Nerus, Malaysia.

E-mail:nurulhikmah@unisza.edu.my
}

Consequently, other alternatives for immunomodulatory agents from other sources, such as natural products, garner more interest among researchers. This is because the specific agents have the potential to elicit the normal body's immune defense system to provide improved protection against microbial infection. Moreover, immunomodulatory therapies also provide benefits by targeting the host rather than the specific pathogen, which later would reduce the incidence of microbial resistance (Relman and Lipsitch, 2018).

Macrophages have gained great interest within the previous decade and currently their role in the stimulation of innate immunity to potentially prevent IDs is getting appreciated (Gupta et al., 2017). Macrophages or mononuclear phagocytes are majorly found in connective tissues and every organ in the body and these cells are also well known as professional phagocytes in which they express a multitude of receptors on their surfaces (Mitchell and 
Isberg, 2017; Murray and Wynn, 2012). Phagocytosis is the main physiological process of macrophages in innate immunity. It is apparent that phagocytosis plays an important role in contributing to the effectiveness of macrophages in the inflammatory responses toward pathogens (Richards and Endres, 2016). Besides, the inflammatory mediators released during phagocytosis, such as reactive nitrogen species (RNS) and reactive oxygen species produced by the activated macrophages, provide the first line of defense toward infections (Kavanthas et al., 2019; Mittal et al., 2014). RNS consists of nitric oxide (NO) which reacts with superoxide $\left(\mathrm{O}_{2}^{-}\right)$to form peroxynitrite $\left(\mathrm{ONOO}^{-} / \mathrm{ONOOH}\right)$ (Grainger et al., 2017; Predonzani et al., 2015). Despite numerous other inflammatory mediators produced by macrophages, NO has been identified as one of the vital players involved in the innate immune response of this cell (Predonzani et al., 2015). It has been well documented that the production of $\mathrm{NO}$ is associated with inducible nitric oxide synthase (iNOS) expression (Mao et al., 2015).

Centella asiatica, which belongs to the Umbelliferae family, is a creeping, perennial, and slender trailing herb. Centella asiatica can be found almost all over the world. It is a native plant to warmer region countries of both the north and south hemispheres, including Asia, Africa, India, Southern Australia, Venezuela, and Madagascar (Jahan et al., 2012). The pentacyclic triterpenes constitute the main active constituents of $C$. asiatica that include madecassoside (MA), asiaticoside (saponin glycosides), and their sapogenins which include asiatic acid (AA) and madecassic acid (Hashim et al., 2011; Roy et al., 2011). The finding by Puttarak and Panichayupakaranant (2012) demonstrated that the highest amounts of AA and MA in $C$. asiatica were taken from five areas in Thailand as compared with other pentacyclic triterpenes. Moreover, the study by Zainol et al. (2008) on the profiling of $C$. asiatica leaves extract from Malaysia exhibited the highest concentrations of AA and MA in leaves which were at the amounts of $1,142.67$ and $5.30 \mu \mathrm{g} / \mathrm{ml}$, respectively, as compared to the other bioactive compounds. Based on numerous previous studies, the pentacyclic triterpenes derived from plants presented significant immunomodulatory properties (Harun et al., 2019, Harun et al., 2020). Approximately $8 \%$ of the dry mass of $C$. asiatica contains these compounds. Most of them are concentrated in leaves and the amounts differ based on the geographical distribution, growth conditions, and genetic and environmental factors (Gray et al., 2018). As far as our literature survey on the previous immunomodulatory studies of $C$. asiatica, there has been no research conducted on the effects of AA and MA alone and in combination on the phagocytosis activity of normal macrophage cell lines (J774A.1). Therefore, recognizing the importance of phagocytosis activity in the innate immunity of macrophages, this present study determines the effects of these compounds on phagocytic uptake, NO production, and iNOS expression of macrophages.

\section{MATERIALS AND METHODS}

\section{Reagents and materials}

The reagents and materials used in this study included Dulbecco's modified eagle medium (DMEM) (Gibco), fetal bovine serum (FBS), penicillin-streptomycin (Gibco), Griess Reagent Kit (Promega, USA), iNOS IgG2a mouse monoclonal antibody
(Becton Dickinson, USA), $\beta$-actin IgG1 mouse monoclonal antibody (Cell Signaling Technologies, USA), secondary antibody goat anti-mouse $\mathrm{IgG}$, horseradish peroxidase (HRP) conjugate (Invitrogen, USA), and Chemi-Lumi One Super Kit (Nacalai Tesque, Japan). All other reagents and chemicals were of the purest commercial grade available.

\section{Plant materials}

The AA compound with purity more than $98 \%$ and MA (purity $\geq 95 \%$ ) assessed using high-performance liquid chromatography derived from C. asiatica in stock of $10 \mathrm{mg} /$ vial were purchased from Sigma-Aldrich with product numbers A2612 and M6949, respectively. Both compounds were stored at $2^{\circ} \mathrm{C}-8^{\circ} \mathrm{C}$. The dilution of both compounds was freshly prepared for each assay using dimethyl sulfoxide (DMSO) and media solutions and the final concentration of DMSO was adjusted to less than $0.1 \%(\mathrm{v} / \mathrm{v})$ to eliminate the cytotoxicity effect by the solvent. Based on our previous cytotoxic assessment (Harun et al., 2018), the concentrations of AA and MA alone used in this study are $0.78,6.25$, and $50 \mu \mathrm{g} / \mathrm{ml}$. The combination treatment used their half dosage of each compound alone (50:50) and were prepared in equal volumes (AA + MA: $0.39+0.39,3.13+3.13$, and $25+25$ $\mu \mathrm{g} / \mathrm{ml}$ ) (Harun et al., 2018). The chemical structures of AA and MA are illustrated in Figure 1.
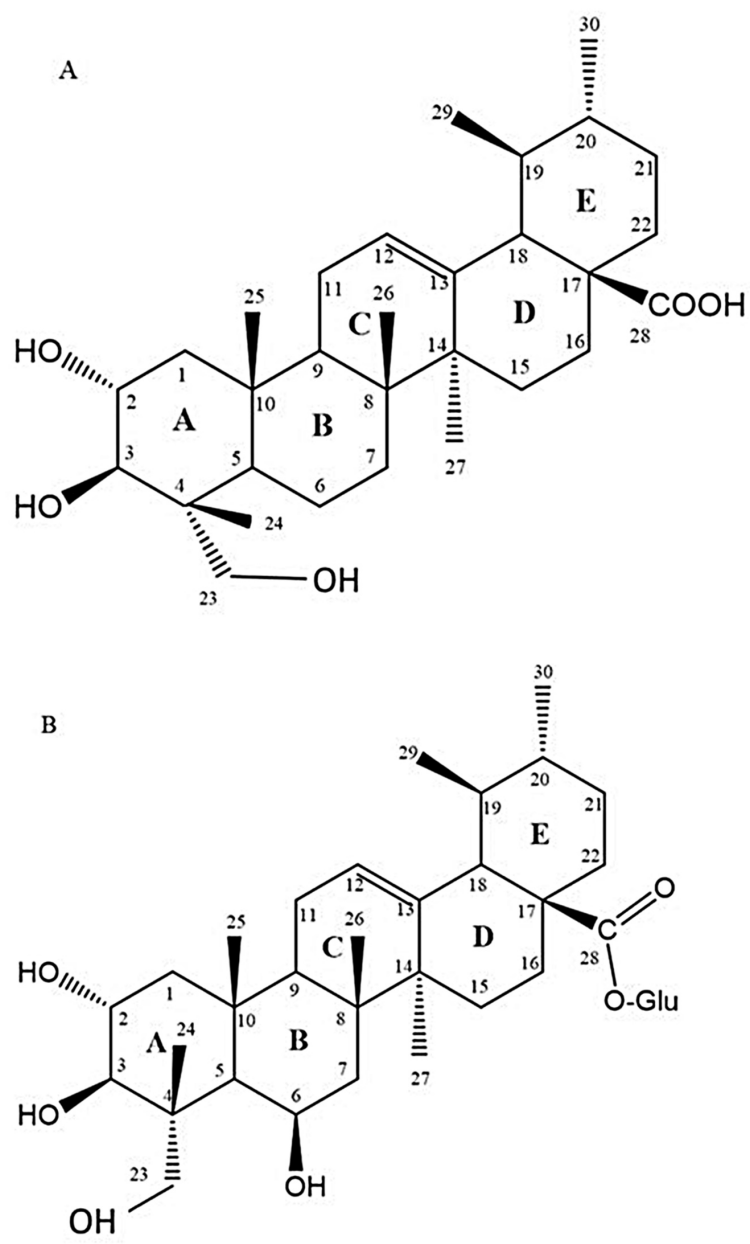

Figure 1. Chemical structure of A) asiatic acid (AA) and B) madecassoside (MA). 


\section{Cell culture maintenance and treatment}

Mouse macrophages cell line J774A.1 (code number ATCC\#TIB-67) was purchased from the American Type Culture Collection (ATCC, USA). The cell culture procedure conducted in this study involved a standard cell culture technique as provided in a good cell culture practice guideline (Coecke et al., 2005). All the techniques comprising cell culture and maintenance were carried out by using an aseptic technique and in a sterile condition. This cell was cultured in a complete medium containing fresh DMEM supplemented with $10 \%$ of heat-inactivated FBS and $1 \%$ penicillinstreptomycin $(100 \mathrm{U} / \mathrm{ml})$. Cells were maintained in an incubator at $37^{\circ} \mathrm{C}$ in a humidified environment supplemented with $5 \%$ carbon dioxide $\left(\mathrm{CO}_{2}\right)$ The macrophages at a density of $2 \times 10^{5}$ cells $/ \mathrm{ml}$ were subjected to treatment with all treatment groups. This study comprises three treatment groups, (1) AA-treated groups (50, 6.25, and $0.78 \mu \mathrm{g} / \mathrm{ml})$, (2) MA-treated groups $(50,6.25$, and $0.78 \mu \mathrm{g} /$ $\mathrm{ml}$ ), and (3) a combination of AA- and MA-treated groups (AA + MA: $25+25,3.13+3.13$, and $0.39+0.39 \mu \mathrm{g} / \mathrm{ml}$ ), as well as two control groups, (1) positive control [lipopolysaccharides (LPS) $100 \mathrm{ng} / \mathrm{ml}$ ] and (2) untreated control (0.05\% DMSO). Each sample collection and bioassay was conducted after a 24-hour incubation phase to assess immunomodulatory enhancing properties based on the measured parameters (Choi et al., 2018; Hu et al., 2019).

\section{Analysis of phagocytosis activity}

The phagocytosis activity of macrophages was determined based on the procedure as proposed by Permanasari et al. (2017) with slight modifications. In this assay, C. albicans was used as a test microorganism to evaluate the ability of treated macrophages to phagocytose the microorganism. Candida albicans $2 \times 10^{6}$ cells $/ \mathrm{ml}$ were used for co-culture with macrophages based on the ratio of yeast to macrophage (10:1) to 1 hour. The choice of C. albicans in this part of the study is because of this microorganism that is able to stimulate the macrophages through toll-like receptor (TLR) as comparable as LPS action (Wang et al., 2019). Then, the Giemsa staining protocol was carried out and the images of the phagocytosed cells were captured by using an image analyzer. Calculation of phagocytosis percentage and index was based on the following formulas (More and Pai, 2017):

$$
\begin{aligned}
& \text { Phagocytosis number of macrophages showing }
\end{aligned}
$$

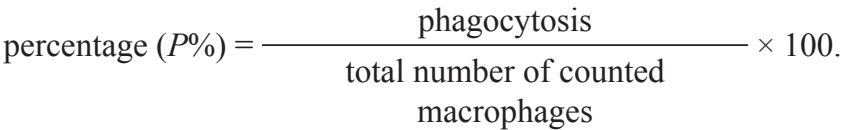$$
\begin{aligned}
& \text { Phagocytosis } \\
& \text { index }(\mathrm{PI})=\frac{\text { total number of yeast cells }}{\text { phagocytosed }} \\
& \begin{array}{c}
\text { number of macrophages showing } \\
\text { phagocytosis }
\end{array}
\end{aligned} \mathrm{P \%} \text {. }
$$

\section{Griess assay}

Nitric oxide which was secreted in the cultures by activated macrophages during treatments was determined by the Griess Reagent Kit (Promega, USA) as described by Haque et al. (2019) with slight modifications. Initially, the cultures of treated, untreated, and control macrophages were collected at 24 hours post treatment and then centrifuged at $300 \times g$ at $4^{\circ} \mathrm{C}$ for 5 minutes. Then, the supernatant of culture for each group of treatment was collected and further aliquoted into $1.5 \mathrm{ml}$ microcentrifuge tubes. All the supernatant samples were kept on ice to avoid protein degradation before proceeding to the Griess assay. The remaining supernatant was stored at $-80^{\circ} \mathrm{C}$ for future use. The standard curve was prepared to measure NO levels in each experimental sample. The nitric standard provided in the kit was prepared by using serial dilution according to the guidelines provided by the manufacturer. Then, $50 \mu \mathrm{l}$ of each supernatant sample was added to a well in triplicate. Next, $50 \mu \mathrm{l}$ of a sulfanilamide solution was dispensed into each well that contained supernatant samples and dilution series of nitric standard solutions, followed by incubation for 10 minutes at room temperature $\left(27^{\circ} \mathrm{C}\right)$ in a dark environment. After that, a similar amount of $N$-(1-naphthyl)ethylenediamine solution was added to each well and further incubated for another 10 minutes and the plate was protected from any light exposure. Finally, the absorbance was evaluated at $540 \mathrm{~nm}$ using an enzymelinked immunosorbent assay (ELISA) microplate reader. The NO concentration for each sample was determined using the generated nitric standard curve.

\section{Western blot analysis}

The expression of iNOS was further determined by Western blot analysis. The Western blotting technique was carried out based on Haque et al. (2019) and Hui et al. (2019), with slight modifications. Briefly, after a 24-hour incubation period, the total protein for each treatment group was extracted from macrophages. $20 \mu \mathrm{g}$ of total protein was separated through sodium dodecyl sulfate-polyacrylamide gel electrophoresis comprising $10 \%$ resolving gel and 5\% stacking gel. The trapped protein was then transferred onto a polyvinylidene difluoride membrane and blocked in $50 \mathrm{ml}$ of blocking buffer solution (1\% skim milk in Tris-buffered saline-Tween 20 (TBST20) for 1 hour at room temperature with gentle shaking. Next, after the washing step, the membrane was incubated with a specific primary antibody solution for iNOS (Becton Dickinson, USA) and $\beta$-actin (Cell Signaling Technologies, USA) mouse monoclonal antibody with a dilution of 1:1,000 (with blocking buffer) overnight at $4^{\circ} \mathrm{C}$ with gentle agitation on the laboratory rocker-shaker. After washing, the membrane was incubated with secondary antibody goat antimouse IgG and HRP conjugate with a dilution of 1:5,000 for 2 hours at room temperature and immunodetection was carried out using the enhanced chemiluminescence method using the ChemiLumi One Super Kit (Nacalai Tesque, Japan). $\beta$-actin was used as an internal control.

\section{Statistical analysis}

Analysis of data was conducted using Statistical Package for the Social Sciences version 23.0. Each sample was measured in triplicate in three independent experiments. The data were presented as mean \pm standard error of the mean (SEM) and the values were obtained from at least three determinations. All the data were analyzed using the one-way analysis of variance and Tukey's post-hoc test for multiple comparisons. All tests were two-tailed and the significance level was set at the least $p$ $<0.05$. 


\section{RESULTS}

The combination ratio selection is based on the concentration of a single compound that gives no toxic effect on macrophages as shown in Harun et al. (2018). Briefly, the optimization of the effects of the combination of AA and MA (\%) $(25: 75,75: 25,50: 50$, and 100:100) on the viability of macrophages was determined using the MTT assay (Guo et al., 2012). Next, the combination ratio was chosen based on the ratio $(50: 50)$ that produces the highest macrophage proliferation, as shown in Figure 2 , and further applied to other subsequent immunological assays. The ratio dosage of the combination compounds was preferred to be lower than the individual compound because the aim of this study is to increase the functional activities of the combined treatment along with the reducing concentration of each single compound (Guo et al., 2012).

Representative pictures of phagocytosis activity of macrophages of each treatment group and the phagocytic index (PI) of the macrophages are shown in Figures 3 and 4, respectively. As shown in Figure 3, the morphology of the uninfected macrophages maintained the normal sphere shape, whereas untreated, LPSstimulated, single, and mixed AA- and MA-treated macrophages incubated with $C$. albicans produced many vacuoles inside their cells known as phagolysosomes to store the phagocytosed pathogens. Macrophages treated with $100 \mathrm{ng} / \mathrm{ml}$ LPS as a positive control presented the highest phagocytic uptake of $C$. albicans as compared to the untreated cells and other groups of treatment. Equally, the macrophages treated with the combination treatments of AA and MA $(0.39+0.39,3.13+3.13$, and $25+25 \mu \mathrm{g} / \mathrm{ml})$ also phagocytosed a higher number of $C$. albicans as compared to the untreated macrophages and macrophages treated with AA and MA alone at their respective concentrations $(0.78,6.25$, and $50 \mu \mathrm{g} /$ $\mathrm{ml})$. These increment values were in a dose-dependent manner. Similarly, with combination treatment, higher intracellular numbers of $C$. albicans were also observed in single MA-treated macrophages when the concentration increased. On the other hand, single AA treatment at all tested concentrations only increased the uptake of small numbers of C. albicans by macrophages.

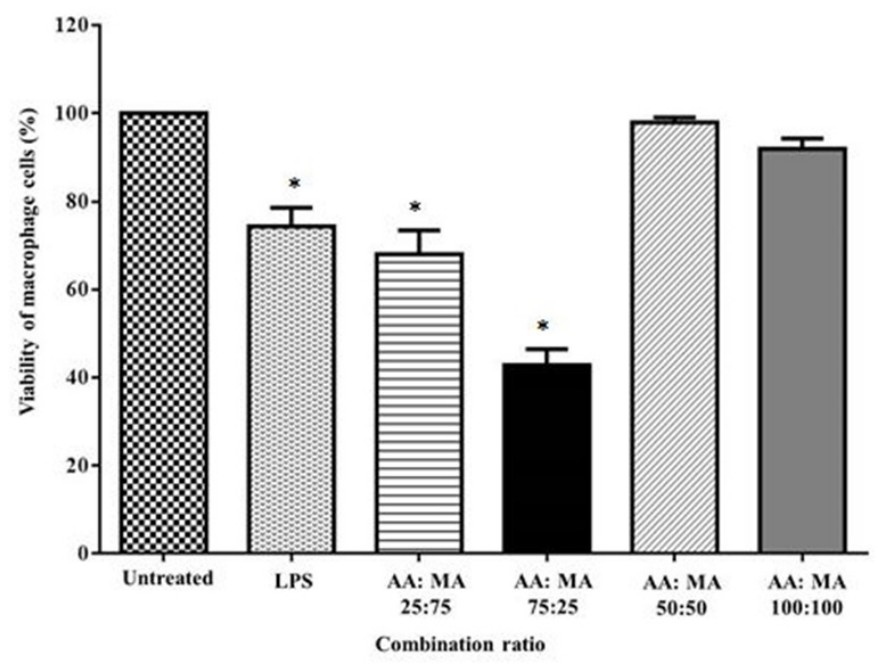

Figure 2. Optimization of combination ratio effects of AA and MA on the viability percentage of macrophages after 24 hours of treatment $(n=3)$. ${ }^{*} p<$ 0.05 when compared to the untreated group.
As shown in Figure 4, the macrophages produced the highest PI $(10.53 \pm 0.3132)$ in response to LPS. For the treatment groups, the highest and most significant PI was observed in macrophages treated with a combination of AA and MA $(25+25$ $\mu \mathrm{g} / \mathrm{ml}$ ) with a PI value $7.117 \pm 0.1035$ followed by $3.13+3.13$ $\mu \mathrm{g} / \mathrm{ml}$ with a PI value $5.907 \pm 0.07424$ when compared to the untreated cells $(1.570 \pm 0.03606)(p<0.001)$. These treatments also promoted significant enhancement in phagocytosis activities of macrophages when compared to the macrophages treated with AA and MA alone at respective dosages (50 and $6.25 \mu \mathrm{g}$ / ml) $(p<0.0001)$. As relative to the untreated macrophages, only macrophages treated with MA alone at doses of 6.25 and $50 \mu \mathrm{g} /$ $\mathrm{ml}$ significantly increased the phagocytic activity of macrophages with the PI values of $2.8 \pm 0.1323(p<0.01)$ and $3.220 \pm 0.1710$ $(p<0.0001)$, respectively. Unpredictably, higher phagocytosis activities of macrophages were recorded after treatment with the two highest concentrations of MA alone when compared to the macrophages incubated with the combination treatment (AA + MA) at the lowest concentration $(0.39+0.39 \mu \mathrm{g} / \mathrm{ml})$. On the contrary, no significant difference in phagocytic activity was observed in macrophages treated with all tested concentrations of AA $(0.78,6.25$, and $50 \mu \mathrm{g} / \mathrm{ml})$.

The effects of AA and MA alone and in combination on NO production of macrophages after 24 hours of treatment are presented in Figure 5. The findings showed that all the
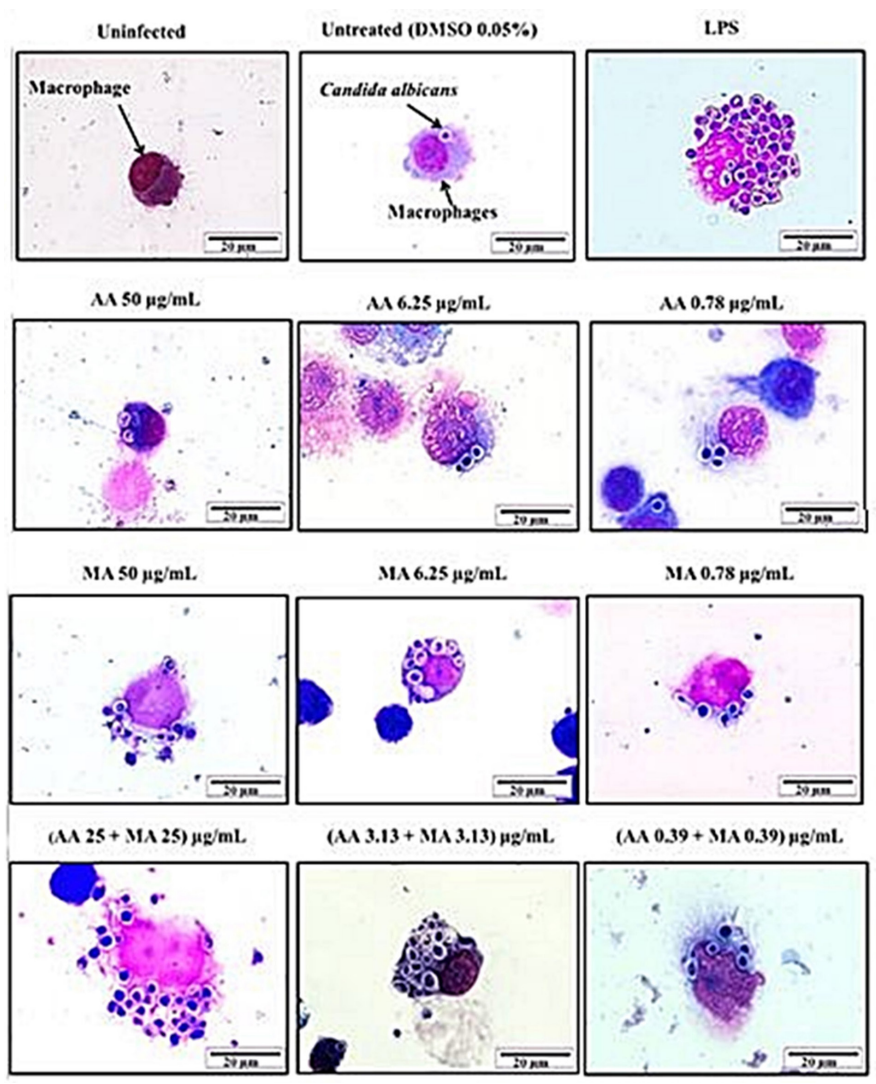

Figure 3. Representative of phagocytic uptake of $C$. albicans by treated macrophages (J774A.1) with individual AA and MA and their combination for 24 hours of treatment. Untreated and LPS-stimulated cells $(100 \mathrm{ng} / \mathrm{ml})$ were used as negative and positive controls, respectively. Observation was made under $1,000 \times$ total magnification of an inverted microscope $(n=3)$. 


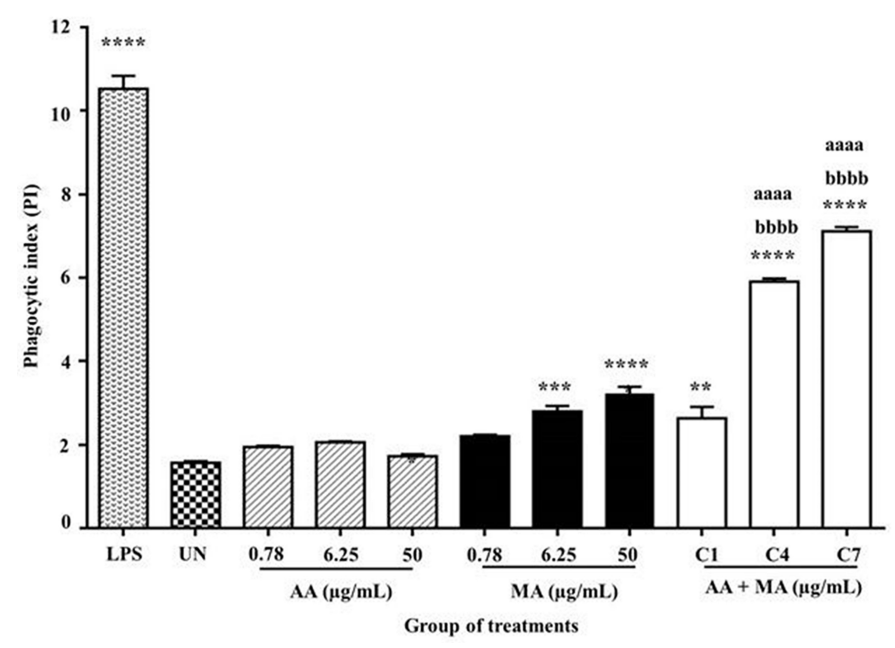

Figure 4. Effects of individual AA and MA and their combination on PI of mouse macrophages (J774A.1) treated with individual AA and MA and their combination for 24 hours and followed co-culture with $C$. albicans for 1 hour. The values are presented as mean $\pm \operatorname{SEM}(n=3) .(\mathrm{C} 1: 0.39+0.39, \mathrm{C} 4: 3.13+$ 3.13, and C7: $25+25) .{ }^{*} p<0.05,{ }^{*} p p<0.01, * * * p<0.001$, and $* * * * p<0.0001$ when compared to the untreated group. ${ }^{\mathrm{a}} p<0.05$, ${ }^{\text {aa }} p<0.01$, ${ }^{\text {aaa }} p<0.001$, and ${ }^{\text {aaaa }} p$ $<0.0001$ when compared to individual AA and ${ }^{\mathrm{b}} p<0.05 .{ }^{\mathrm{bb}} p<0.01,{ }^{\mathrm{bbb}} p<0.001$, and ${ }^{\text {bbbb }} p<0.0001$ when compared to individual MA.

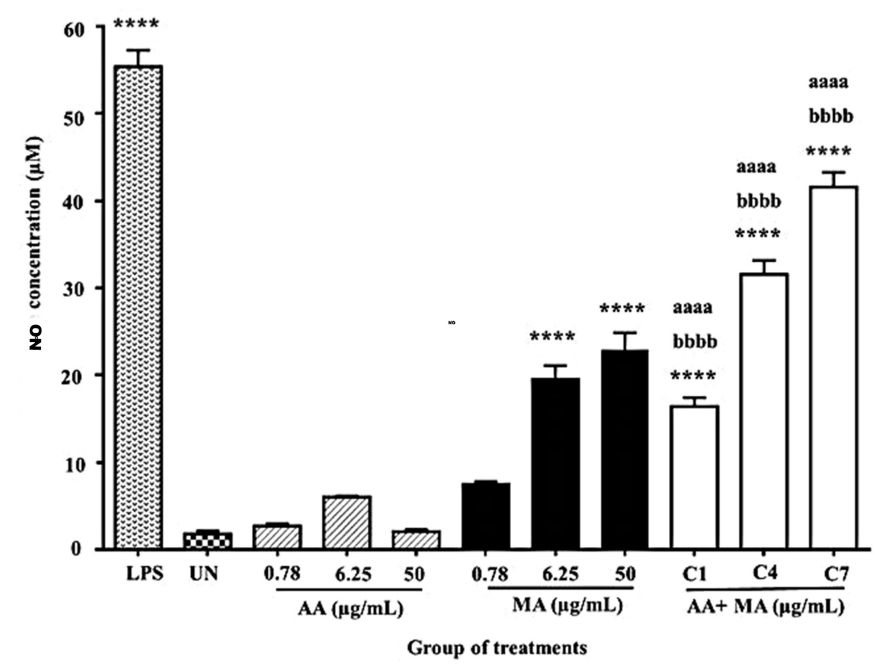

Figure 5. Effects of individual AA and MA and their combination treatments on the NO production of mouse macrophages (J774A.1) for 24 hours $(n=3)$. $(\mathrm{C} 1$ : $0.39+0.39, \mathrm{C} 4: 3.13+3.13$, and C $7: 25+25) . * p<0.05, * * p<0.01, * * * p<$ 0.001 , and $* * * * p<0.0001$ when compared to the untreated group. ${ }^{a} p<0.05$, ${ }^{\text {aa }} p<0.01$, ${ }^{\text {aaa }} p<0.001$, and ${ }^{\text {aaaa }} p<0.0001$ when compared to individual AA. ${ }^{\text {b }} p$ $<0.05,{ }^{\mathrm{bb}} p<0.01,{ }^{\mathrm{bbb}} p<0.001$, and ${ }^{\mathrm{bbbb}} p<0.0001$ when compared to individual MA.

combination treatments (AA + MA) at every dosage $(0.39+$ $0.39,3.13+3.13$, and $25+25 \mu \mathrm{g} / \mathrm{ml})$ significantly elevated $\mathrm{NO}$ production of macrophage cells in a concentration-dependent manner with values of $16.42 \pm 1.026,31.57 \pm 1.581$, and 41.62 $\pm 1.658 \mu \mathrm{M}$, respectively, after 24 hours when compared to the untreated macrophages $(1.790 \pm 0.3330 \mu \mathrm{M})(p<0.0001)$. Similar significant results were shown when all the data were compared to the NO production of the macrophages treated with AA and MA alone at the comparative concentrations $(0.78,6.25$, and 50

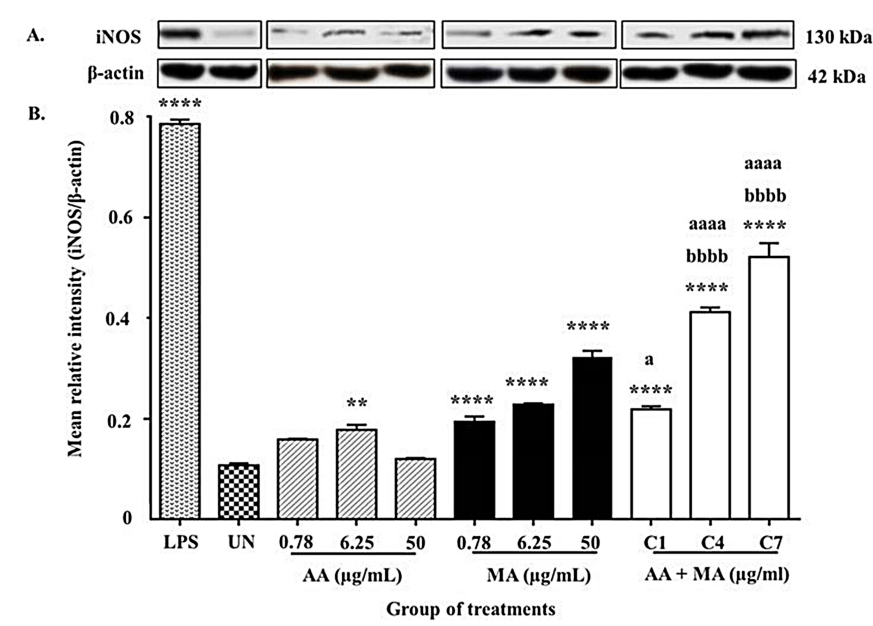

Figure 6. (A) Representative results of iNOS and $\beta$-actin proteins expression. (B) The relative density of iNOS/ $\beta$-actin proteins expression in macrophages treated with individual AA and MA and their combination for 24 hours $(n=3)$. $(\mathrm{C} 1: 0.39+0.39, \mathrm{C} 4: 3.13+3.13$, and C7: $25+25) .{ }^{*} p<0.05, * * p<0.01,{ }^{*} * * p$ $<0.001$, and $* * * * p<0.0001$ when compared to the untreated group. ${ }^{a} p<0.05$, ${ }^{\text {aa }} p<0.01$, ${ }^{\text {aaa }} p<0.001$, and aaa $p<0.0001$ when compared to individual AA. ${ }^{\text {b }} p$ $<0.05,{ }^{\mathrm{bb}} p<0.01,{ }^{\mathrm{bbb}} p<0.001$, and ${ }^{\mathrm{bbbb}} p<0.0001$ when compared to individual MA.

$\mu \mathrm{g} / \mathrm{ml})(p<0.0001)$. As expected, a similar pattern was shown by macrophages treated with LPS at $100 \mathrm{ng} / \mathrm{ml}$ which significantly boosted NO production to $55.40 \pm 1.875 \mu \mathrm{M}$ when compared to the untreated macrophages $(p<0.0001)$.

Additionally, there was also significant stimulation of NO production by macrophages treated with MA alone at both concentrations of $6.25 \mu \mathrm{g} / \mathrm{ml}(19.55 \pm 1.565 \mu \mathrm{M})$ and $50 \mu \mathrm{g} / \mathrm{ml}$ $(22.89 \pm 1.969 \mu \mathrm{M})(p<0.0001)$. Unpredictably, the two highest concentrations of MA alone promoted higher production of NO of macrophages when compared to the effect of the lowest combination dosage $(0.39+0.39 \mu \mathrm{g} / \mathrm{ml})$ used in this study. On the contrary, the treatments with the lowest dosage of MA alone $(0.78$ $\mu \mathrm{g} / \mathrm{ml})$, as well as AA at three tested concentrations $(0.78,6.25$, and $50 \mu \mathrm{g} / \mathrm{ml}$ ), did not significantly enhance the NO production after 24 hours of the incubation period.

To verify whether the upsurge of NO secretion by compound treatment was due to the enhancement of iNOS expression, the effects of AA and MA alone and in combination on iNOS expression were measured. Figure 6A shows the expression of a band at approximately $130 \mathrm{kDa}$ (the expected size of iNOS) which was detected in the Western blot analysis of macrophages in all groups of compound treatments, the untreated group, and the positive control group. The MRI of iNOS against the $\beta$-actin value was used to predict the expression of iNOS in macrophages as shown in Figure 6B.

As shown in Figure B, the overall findings showed a similar equivalent pattern with NO production outcome. When a comparison was made between these groups, the combination treatment $(\mathrm{AA}+\mathrm{MA})$ at concentrations of $0.78+0.78,3.13+$ 3.13 , and $25+25 \mu \mathrm{g} / \mathrm{ml}$ carried out significant stimulatory activity in iNOS expression represented with MRI values of $0.5213 \pm 0.02738$ and $0.4113 \pm 0.00933$, correspondingly when compared to the untreated group $(0.1073 \pm 0.00426 \mathrm{MRI})(p<$ $0.0001)$. However, only two concentrations $(3.13+3.13$ and $25+$ 
$25 \mu \mathrm{g} / \mathrm{ml}$ ) from these combination treatments exhibited significant enhancement in iNOS expression when compared with both AA and MA alone at their respective dosages $(p<0.0001)$. The effects took place in a dose-dependent manner and were represented with high-intensity bands. The comparable effect of the combination treatments was also shown by the macrophages treated with LPS with the value of $0.7857 \pm 0.007965$ MRI. On the contrary, the combination treatment $(\mathrm{AA}+\mathrm{MA})$ at the lowest concentration $(0.39+0.39 \mu \mathrm{g} / \mathrm{ml})$ only presented significant augmentation in iNOS expression of macrophages but it was minimal when compared to the treatment of AA alone $(0.78 \mu \mathrm{g} / \mu \mathrm{g} / \mathrm{ml})(p<0.05)$.

Similarly, the treatment of MA alone also significantly increased the expression of iNOS of macrophages for all the tested concentrations of treatments $(0.78,6.25$, and $50 \mu \mathrm{g} / \mathrm{ml})$ when compared to the untreated control group $(p<0.0001)$. Nevertheless, the significant upsurge in iNOS expression was only exhibited in the macrophages supplied with AA alone at the dosage of $6.25 \mu \mathrm{g} / \mathrm{ml}$ as relative to the untreated macrophages ( $p$ $<0.01)$. The overall findings displayed that the elevation of iNOS expression was represented by high band intensity.

\section{DISCUSSION}

In an effort to evaluate the plant compounds-induced modulation of innate immune responses of macrophages, this study determined the effects on phagocytosis activity. This part of the study was conducted by measuring the uptake of killed $C$. albicans (in a duration of 1 hour) by macrophages after being treated with single and mixed compounds for 24 hours. Candida albicans was used as an example of pathogenic microorganisms which are normally ubiquitous in the environment and based on the established method to evaluate the immunomodulatory potential of natural products (Sumithra et al., 2017). The upsurge in phagocytosis activity is accepted as one of the most distinct properties of macrophages activation and contributes to the stimulation of innate immune responses (Gordon, 2016).

The findings showed that the phagocytic activity of macrophages markedly elevated when stimulated with combination treatments $(\mathrm{AA}+\mathrm{MA})$ at the concentrations of $25+$ 25 and $3.13+3.13 \mu \mathrm{g} / \mathrm{ml}$ and was represented by higher uptake of $C$. albicans as compared to the other compound-treated and untreated macrophages. The findings in this study have supported the earlier preliminary study by Mali and Hatapakki (2008) who identified that various concentrations of ethanolic leaves extract of C. asiatica $(25,50$, and $100 \mathrm{mg} / \mathrm{ml})$ significantly increased the PI and intracellular killing properties of neutrophils when compared to the untreated group by using the slide method and qualitative nitroblue tetrazolium test. Moreover, the phagocytosis results also are in line with the other in vivo investigation by Besung (2009) who used mice infected with Salmonella typhi as a model. This study presented that the methanolic extract of $C$. asiatica leaves $(125,250$, and $500 \mathrm{mg} / \mathrm{kg} \mathrm{BW})$ enhanced the phagocytic capacity of macrophages in a dose-response relationship with the highest phagocytic value of macrophages observed in treated mice (500 $\mathrm{mg} / \mathrm{kg} \mathrm{BW}$ ) when compared with untreated mice.

The result of the uptake of $C$. albicans by macrophages during phagocytosis is also comparable with the finding by Besung et al. (2012). In detail, through their observation of the Giemsastained cells under an inverted microscope, the team demonstrated that the $C$. asiatica extract-treated macrophages displayed higher uptake of $S$. typhi with the increment of extract concentration. In addition to that, the treated macrophages presented with irregular shapes, eccentric location of the horseshoe-shaped nucleus, and a lot of vesicles and vacuoles of lysosomes when compared to the morphology of untreated macrophages (appeared small and with regular circular shapes). Furthermore, earlier research conducted by Gupta et al. (2014) also proved that the crude extract of $C$. asiatica is comprised of triterpene components, especially AA and MA. Hence, our results supported previous findings showing that the increment of the phagocytosis activities could be due to the effects of AA and MA.

NO is a vital molecule involved in several fundamental and pathologic developments. The NO production by macrophages is crucial for the mammalian immune defense mainly in innate immunity. Accordingly, NO can be used as a measurable index of macrophage stimulation (Grainger et al., 2017). The production of NO and its synthesized protein, iNOS, is believed to be reliant on the secretion of proinflammatory cytokines (Venkatesan et al., 2017). Thus, this study considered the effect of treatment on the production of NO which was also measured in the supernatant acquired from the culture of macrophages in all treatment groups. From the analyzed data, it was found that all the combination treatments resulted in an increase and higher NO production at all the ratios of tested combination dosages as compared to the untreated group. This finding was countered by Jeong et al. (2002) and You et al. (2001) in their in vivo studies which discovered the immunomodulatory effects of $\alpha$-hederin and ursolic acid, respectively. Both compounds in the group of triterpene saponin have been shown to elicit NO production of harvested peritoneal macrophages from treated mice in a concentrationdependent manner. Furthermore, other previous in vitro studies also revealed that other group compounds such as water-soluble polysaccharides from tiger lilies at the concentration of 25-400 $\mu \mathrm{g} / \mathrm{ml}$ also dose-dependently stimulated the phagocytosis activity and NO production of macrophages RAW 264.7 (Chen et al., 2014). Additionally, in another in vitro research finding, a similar indication was established in which a fraction from Laminaria japonica containing several bioactive constituents exhibited significant enhancement of the production of NO in macrophages with the highest value being $20.4 \mu \mathrm{mol} / 1$ when treated with this fraction at the concentration of $100 \mu \mathrm{g} / \mathrm{ml}$ (Fang et al., 2015).

Western blot analysis was conducted to investigate whether the stimulation of $\mathrm{NO}$ production is associated with its iNOS protein. The increase of NO secretion was related to the increase of its iNOS expression with greater significant enhancement of iNOS expression being observed in macrophages treated with combination treatment (AA + MA) at dosages of $25+25$ and 3.13 $+3.13 \mu \mathrm{g} / \mathrm{ml}$ as compared to the untreated control group. All these treatments also presented similar significant augmentation in iNOS protein as relative to both single treatments. The finding of this study is also consistent with the observations by Lee et al. (2010) who demonstrated that the pyruvylated sulfated galactan compound from Codium fragile stimulated NO production as well as iNOS expression in macrophages RAW 246.7. In addition, these present research findings are in agreement with another previous study which exhibited that the antitumor potential of polysaccharides derived from Grifola frondosa was related to the ability of this compound to augment NO production and iNOS expression in macrophages RAW 264.7 (Mao et al., 2015). Nevertheless, based on the literature, there is still a lack of studies on the effects of a combination of plant 
compounds in immunostimulating macrophages and other immune cells. Therefore, the specific comparison of this current finding to previous studies is only conducted relative to the research conducted using crude extracts and a single compound as a treatment.

The outcomes also exhibited that the NO only was produced at the basal line by untreated macrophages and was comparable with its iNOS expression. It is also similar in the normal state of tissue such as mucosa of colon, mRNA, and protein expression of iNOS in resident macrophages which was presented at a low level (Wallace et al., 2019). However, when the pathogenic microorganisms such as Salmonella spp., Enterococcus faecalis, Escherichia coli, and Chlamydia psittaci destructed the tissue, they were then recognized by the TLR of macrophages (Behnsen et al., 2015; Lagae and Vanrompay, 2015; Mallick et al., 2019; Xu et al., 2018). The activated macrophages further amplified their iNOS expression at either the mRNA or protein levels by activating the signaling molecules.

According to the previous studies, there is no research conducted to determine the effects of the bioactive compounds derived from $C$. asiatica on normal immune cells to represent a healthy community. There was only a study by Yun et al. (2008) that showed that the AA isolated from $C$. asiatica strongly inhibited LPS-induced NO and PGE2 production in RAW 264.7 macrophages. This compound was more potent than its derivative, namely asiaticoside. By comparing their chemical structures, the presence of glucose at C-28 (ring E) of asiaticoside might reduce its anti-inflammatory effect. Furthermore, AA along with corosolic acid and alphitolic acid has been tested for their anti-inflammatory effect in an animal model. All compounds revealed an inhibition effect in TPA-induced inflammation. However, corosolic acid presented the most potent activities when measured using the arachidonic acid assay. The lack of one alcoholic group at C-24 (ring A) probably contributed to its activity (Aguirre et al., 2006).

In accordance with the immunostimulation activities of combination compounds on the normal model of macrophages, it has been assumed that these activities are initiated from the unique molecular structure of the sugar glucose-glucose-rhamnose at $\mathrm{C}-28$ and hydroxyl $(\mathrm{OH})$ group $(\mathrm{C}-6)$ located on the MA molecular structure. It is essential to highlight the theory that both of these molecules might have interacted with the molecular structure of AA and then enhance the interaction of these molecules with TLR of macrophages which further activates the macrophage cell.

\section{CONCLUSION}

In a nutshell, the combination treatment $(\mathrm{AA}+\mathrm{MA})$ at the maximum concentration $(25+25 \mu \mathrm{g} / \mathrm{ml})$ enhances phagocytosis activity that involves phagocytic uptake of the tested microorganism, NO production, and iNOS expression in normal macrophages after 24 hours of treatment. The finding suggests that the combination treatment is able to cause an upsurge of innate immune responses of macrophages and might be used for future benefits to stimulate the immune system as a preventive agent against potential infection.

\section{ACKNOWLEDGMENTS}

The authors gratefully acknowledge the use of services and facilities at the School of Health Sciences (PPSK) and School of Dental Sciences (PPSG) of the University of Science Malaysia (USM). This study was funded by a USM bridging grant (304. PPSK.6316150). The authors also want to thank Ms. Nor Munirah Zakaria for her help and guidance in cell culture maintenance.

\section{CONFLICTS OF INTEREST}

The authors report no financial or any other conflicts of interest in this work.

\section{ETHICAL APPROVALS}

This study does not involve experiments on animals or human subjects.

\section{REFERENCES}

Aguirre MC, Delporte C, Backhouse N, Erazo S, Letelier ME, Cassels BK, Silva, X, Alegria S, Negrete R. Topical anti-inflammatory activity of $2 \alpha$-hydroxy pentacyclic triterpene acids from the leaves of Ugni molinae. Bioorg Med Chem, 2006; 14(16):5673-7.

Behnsen J, Perez-lopez A, Nuccio S, Raffatellu, M. Exploiting host immunity : the Salmonella paradigm. Trends Immunol, 2015; 36:112-20.

Besung INK. Pegagan (Centella asiatica) sebagai alternatif pencegahan penyakit infeksi pada ternak. Bull Vet Udayana, 2009; 1:61-7.

Besung, I, Astawa, N, Suatha, I, Hartangningsih. Centella asiatica extract increased on the level of interleukin 6 (IL-6) in mice. Indones J Biomed Sci, 2012; 5(1):1-7.

Chee WA, Angie MJ, Matthew A. Nitroimidazoles: molecular fireworks that combat a broad spectrum of infectious diseases. J Med Chem, 2017; 60(18):7636-57.

Chen ZG, Zhang DN, Zhu Q, Yang QH, Han YB. Purification, preliminary characterization and in vitro immunomodulatory activity of tiger lily polysaccharide. Carbohydr Polym, 2014; 106:217-22.

Choi EY, Jin JY, Hyeon JY, Choe SH, Keum BR, Lim JM, Cho, KK, Choi IS. Immunomodulatory activity of commercial macrophage cell line RAW 264.7. J Environ Biol, 2018; 39:166-74.

Coecke S, Balls M, Bowe G, Davis J, Gstraunthaler G, Hay R, Merten O, Price A, Schechtman L. Guidance on good cell culture practice a report of the second ECVAM task force on good cell culture practice. Altern Lab Anim, 2005; 33:261-87.

Fang Q, Wang J, Zha X, Cui S, Cao L, Luo J. Immunomodulatory activity on macrophage of a purified polysaccharide extracted from Laminaria japonica. Carbohydr Polym, 2015; 134:66-73.

Glick M. The good, the bad, and the ugly. J Am Dent Assoc, $2016 ; 147: 771-3$

Gordon S. Phagocytosis: the legacy of Metchnikoff. Cell, 2016; 166(8):1065-8

Grainger JR, Konkel JE, Zangerle-Murray T, Shaw TN Macrophages in gastrointestinal homeostasis and inflammation. Pflugers Arch, 2017; 469:527-39.

Gray NE, Alcazar MA, Lak P, Wright KM, Quinn J, Stevens JF, Maier CS, Soumyanath A. Centella asiatica: phytochemistry and mechanisms of neuroprotection and cognitive enhancement. Phytochem Rev, 2018; 17:161-94.

Guo S, Qiu P, Xu G, Wu X, Dong P, Yang G, Zheng J, Mcclements DJ, Xiao H. Synergistic anti-inflammatory effects of nobiletin and sulforaphane in lipopolysaccharide-stimulated RAW 264.7 cells. J Agric Food Chem, 2012; 60(9):2157-64.

Gupta A, Verma, S, Kushwaha, P, Srivastava, S, Rawat AKS Pharmacognosy quantitative estimation of asiatic acid, asiaticoside \& madecassoside in two accessions of Centella asiatica (L.) Urban for morpho-chemotypic variation. Pharm Res, 2014; 48(3):75-9.

Gupta PK, Rajan MGR, Kulkarni S. Activation of murine macrophages by G1-4A, a polysaccharide from Tinospora cordifolia, in TLR4/ MyD88 dependent manner. Int Immunopharmacol, 2017; 50(6):168-77. 
Haque MA, Ibrahim J, Hemavathy H, Shazliana, G. Standardized extract of Zingiber zerumbet suppresses LPS-induced proinflammatory responses through NF- $\mathrm{KB}$, MAPK and PI3K-Akt signaling pathways in U937 macrophages. Phytomedicine, 2019; 54(2):195-205.

Harun NH, Wan Amir NWA, Rapeah S. The effects of individual and combination of asiatic acid and madecassoside derived from Centella asiatica (Linn.) urban on the viability percentage and morphological changes of mouse macrophage cell line (J774A.1). J Appl Pharm Sci, 2018; 8(11):109-15.

Harun NH, Septama AW, Wan Amir NWA, Rapeah S. The potential of Centella asiatica (Linn.) urban as an anti-microbial and immunomodulator agent: a review. Nat Prod Sci, 2019; 25(2):92-102.

Harun NH, Septama AW, Wan Amir NWA, Rapeah S. Immunomodulatory effects and structure-activity relationship of botanical pentacyclic triterpenes: a review. Chin Herb Med, 2020; 12(2):118-24.

Hashim P, Sidek H, Helan MHM, Sabery A, Palanisamy UD, Ilham M. Triterpene composition and bioactivities of Centella asiatica. Molecules, 2011; 16(2):1310-22.

Hu W, Jiang Y, Xue Q, Sun F, Zhang J, Zhou J Structural characterisation and immunomodulatory activity of a polysaccharide isolated from lotus (Nelumbo nucifera Gaertn) root residues. J Funct Foods, 2019; 60:1-11.

Hui T, Zijing L, Youwei P, Yixi B. Immunomodulatory effects exerted by Poria Cocos polysaccharides via TLR4/TRAF6/NF- $\kappa$ B signaling in vitro and in vivo. Biomed Pharmacother, 2019; 112:108709.

Jahan R, Hossain S, Seraj S, Nasrin D, Khatun Z, Das PR, Islam, M, Ahmed I, Rahmatullah M. Centella asiatica (L.) urb.: ethnomedicinal uses and their scientific validations. Am J Sustain Agric, 2012; 6:261-70.

Jeong HG, Choi CY. Expression of inducible nitric oxide synthase by $\alpha$-Hederin in macrophages. Planta Med, 2002; 68:392-96.

Kavanthas PB, Krause PJ, Ruddle NH. Adaptive immunity: antigen recognition by $\mathrm{T}$ and $\mathrm{B}$ lymphocytes. Immunoepidemiology, 2019:55-74.

Lagae S, Vanrompay D. Innate immune response in avian macrophages elicited by Chlamydia psittaci. VLAAMS Diergeneekundig Tijdschrft, 2015; 84(3):133-41.

Lee JB, Ohta Y, Hayashi K, Hayashi T. Immunostimulating effects of a sulfated galactan from Codium fragile. Carbohydr Res, 2010; 345:1452-4.

Mali, RG, Hatapakki, B. An in vitro study of effect of Centella asiatica on phagocytosis by human neutrophils. Int J Pharm Sci Nanotechnol, 2008; 1(3):297-302.

Mallick S, Das J, Verma J, Mathew S, Maiti TK, Ghosh AS. Role of Escherichia coli endopeptidases and DD-carboxypeptidases in infection and regulation of innate immune response. Microbes Infect, 2019; 21(10):464-74.

Mao GH, Ren Y, Feng WW, Li Q, Wu HY, Jin D, Zhao T, Xu CQ, Yang LQ, Wu XY. Antitumor and immunomodulatory activity of a watersoluble polysaccharide from Grifola frondosa. Carbohydr Polym, 2015; 134:406-12.

Maslow JN. Vaccine development for emerging virulent infectious diseases. Vaccine, 2017; 35:5437-43.

Mitchell G, Isberg RR. Innate immunity to intracellular pathogens: balancing microbial elimination and inflammation. Cell Host Microbe, 2017; 22(2):166-75.

Mittal M, Siddiqui MR, Tran K, Reddy SP, Malik AB. Reactive oxygen species in inflammation and tissue injury. Antioxid Redox Signaling, 2014; 20(7):1126-67.

More P, Pai K. Effect of Tinospora cordifolia (Guduchi) on the phagocytic and pinocytic activity of murine macrophages in vitro. Indian $\mathrm{J}$ Exp Biol, 2017; 55(1):21-6.

Murray PJ, Wynn TA. Protective and pathogenic functions of macrophage subsets. Nat Rev Immunol, 2012; 11:723-37.
Permanasari P, Hertiani T, Yuswanto A. In vitro evaluation of massoia bark essential oil and C-10 massoialactone potency as immunomodulator. J Essent Oil Bear Plants, 2017; 2(2):459-67.

Predonzani A, Calì B, Agnellini AHR, Molon B. Spotlights on immunological effects of reactive nitrogen species: when inflammation says nitric oxide. World J Exp Med, 2015; 5:64-77.

Puttarak P, Panichayupakaranant P. Factors affecting the content of pentacyclic triterpenes in Centella asiatica raw materials. Pharm Biol, 2012; 50(12):1508-12.

Relman DA, Lipsitch M. Microbiome as a tool and a target in the effort to address antimicrobial resistance. Proc Natl Acad Sci, 2018; 115(51):12902-10.

Richards DM, Endres RG. How cells engulf: a review of theoretical approaches to phagocytosis. Proc Natl Acad Sci, 2016; 113(22):6113-8.

Roy DC, Barman SK, Shaik M. Current Updates on Centella asiatica : phytochemistry, pharmacology and traditional uses. Med Plant Res, 2011; 3:20-36.

Sumithra M, Chitra V, Gowri K, Ramaswamy R, Gayathiri K, Derera P. In-vitro immunomodulatory effect of hydroalcoholic leaves extract of Avicennia officinalis. Biosci Biotechnol Res Asia, 2017; 14(3):1117-20.

Venkatesan T, Choi YW, Lee J, Kim YK. Pinus densiflora needle supercritical fluid extract suppresses the expression of pro-inflammatory mediators iNOS, IL- 6 and IL-1 $\beta$, and activation of inflammatory STAT1 and STAT3 signaling proteins in bacterial lipopolysaccharide-challenged murine macrophages. DARU J Pharm Sci, 2017; 25:1-10.

Wallace JL. Nitric oxide in the gastrointestinal tract: opportunities for drug development. Br J Pharmacol, 2019; 176:147-54.

Wang W, Deng Z, Wu H, Zhao Q, Li T, Zhu W, Wang X, Tang L, Wang C, Cui SZ, Xiao H, Chen J. A small secreted protein triggers a TLR2/4-dependent inflammatory response during invasive Candida albicans infection. Nat Commun, 2019; 1015(10):1-14.

WHO. Water related diseases. 2000. Available via https://www. who.int/water_sanitation_health/diseasesrisks/diseases/diarrhoea/en/

$\mathrm{Xu} \mathrm{Z}$, Tong Z, Neelakantan P, Cai Y. Enterococcus faecalis immunoregulates osteoclastogenesis of macrophages. Exp Cell Res, 2018; $362: 152-8$.

You HJ, Choi CY, Kim JY, Park SJ, Hahm KS, Jeong HG. Ursolic acid enhances nitric oxide and tumor necrosis factor- $\alpha$ production via nuclear factor- $\mathrm{\kappa B}$ activation in the resting macrophages. FEBS Lett, 2001; 509:156-60.

Yun KJ, Kim JY, Kim JB, Lee KW, Jeong SY, Park HJ, Jung HJ, Cho YW, Yun K, Lee KT. Inhibition of LPS-induced NO and PGE2 production by siatic acid via NF-kappa B inactivation in RAW 264.7 macrophages: possible involvement of the IKK and MAPK pathways. Int Immunopharmacol, 2008; 8(3):431-41.

Zainol NA, Voo SC, Sarmidi MR, Aziz RA. Profiling of Centella asiatica (L.) urban extract. Malays J Anal Sci, 2008; 12(2):322-7.

How to cite this article:

Harun NH, Ahmad WANW, Suppian R. Immunostimulatory effects of asiatic acid and madecassoside on the phagocytosis activities of macrophages cell line (J774A.1). J Appl Pharm Sci, 2021; 11(11):104-111. 